\title{
Acoustoelectric effects in quantum constrictions
}

\author{
Frank A. Maa $\varnothing^{1}$ and Y. Galperin ${ }^{2,3}$ \\ 1 Institutt for fysikk, The Norwegian University of Science and Technology, NTNU, N-7034 Trondheim, Norway \\ ${ }^{2}$ Fysisk institutt, University of Oslo, P. O. Box 1048 N-0316 Oslo, Norway \\ ${ }^{3}$ A. F. Ioffe Physico-Technical Institute R A S, 194021 St. Petersburg, Russia
}

(November 22, 2017)

\begin{abstract}
A dc current induced in a quantum constriction by a traveling acoustic wave (or by nonequilibrium ballistic phonons) is considered. We show that in many important situations the effect is originated from acoustically-induced scattering between the propagating and reflecting states in the constriction. Two particular regimes corresponding to relatively high and low acoustic frequencies are discussed. In the first regime, the acoustoelectric effect in a smooth constriction can be understood by semi-classical considerations based on local conservation laws. For the low frequency regime, we show that the acousto-conductance is closely related to the zero field conductance. The qualitative considerations are confirmed by numerical calculations both for smooth and abrupt channels.
\end{abstract}

\section{INTRODUCTION}

Recently surface acoustic waves (SAW) [1] 3, as well as non-equilibrium ballistic phonons [4], have been successfully used to study mesoscopic systems with a quasione-dimensional electron gas as quantum point contacts (QPC) and quantum wires (QW). In most cases these systems connect two reservoirs made of a twodimensional electron gas (2DEG). Consequently, these studies were a natural continuation of an extensive work on acoustical studies of 2DEG.

Due to the piezoelectric effect, acoustic waves induce traveling waves of electric field which propagate with the sound velocity $s$. Being concentrated near the surface, these waves are extremely sensitive to the electric properties of the relevant region. In particular, a highly conductive 2DEG can screen out the electric fields and in that way influence the sound velocity. If the electric field is not screened completely, the Joule losses in the 2DEG lead to a pronounced attenuation of the waves. In both situations acoustic waves provide a possibility of probeless measurements of the 2D conductance $G(\omega, \mathbf{q})$ as a function of the sound frequency $\omega$ and wave vector $\mathbf{q}$. The role of screening is determined by the dimensionless ratio $G / s$ (note that $G$ has the dimensionality of the velocity in the CGSE system) which is usually large (see e.g. Ref. (5)). However, the component of $G$ parallel to the surface can be suppressed by a magnetic field and thus made of the same order as the sound velocity. This is the case, in particular, under the conditions of the quantum Hall effect, and several beautiful and instructive experiments were conducted in that regime [6, ]. Many important experiments were performed using ballistic phonons (see, e.g., Ref. [8]) which can be induced by laser beams [9] or by resistive film heaters [4].

Two groups of experiments are usually conducted. In the experiments of the first group, a linear response to the wave is studied. Namely, the variations in the sound velocity and attenuation are registered. The second group of experiments [1]2,7] is concentrated on the studies of the response of the electron gas to the acoustic waves. The quantities which are investigated in these experiments are the dc electric current induced by the waves (the so-called acoustoelectric current), or the dc voltage induced across the sample. These effects are non-linear in the sound amplitude, at small amplitudes they are proportional to the amplitude squared.

The acoustic waves seem very advantageous for studies of quantum constrictions like QPC or QW. Indeed, the screening length in the 2DEG coincides with the Bohr radius, $a_{B}$. Consequently, if the dimensions of a 2D sample are much larger than $a_{B}$ then the (piezoelectric) coupling to phonons is screened out, at least in the absence of a perpendicular magnetic field. However, if the width of the channel is not exceeding $a_{B}$ by too much, the field penetrates the channel. As a result, it is the channel region that contributes to the interaction rather than the $2 \mathrm{D}$ leads where the coupling is screened out. On the other hand, the resistance of the system is also determined by the channel, and it is possible either to monitor its changes under the influence of the waves (the so-called acousto-conductance), or to measure the acoustoelectric current. 10]

Recently, two experiments [1]2] on the induced acoustoelectric current in quantum constrictions revealed several interesting features. In the first paper by Shilton et al. [1] the regime of weak acoustoelectric current was investigated, while the second paper [2] examined the regime of a strong SAW-field. In the large-amplitude regime the induced current was shown to be quantized in units of $e f$, with $e$ being the electronic charge and $f=\omega / 2 \pi$ the wave frequency. This behavior was explained by the authors of Ref. [2] in terms of 'traveling quantum dots' with an integer number of electrons in 
each dot. Thus, during one period an integer number of electrons are carried from one side of the constriction to the other. On the other hand, the weak-field regime displayed a complicated pattern of peaks and dips close to pinch-off and a more regular pattern of oscillations for higher gate voltages. It was noted that the structure of the acoustoelectric current resembled (but did not coincide with) that of the derivative of the zero field conductance with respect to the gate voltage.

The low-amplitude behavior of the acoustoelectric current was discussed in Ref. [11] on the basis of the Boltzmann equation for the electrons interacting with the traveling wave. The theory, which was able to reproduce the main features of the experimental observations, is based on a model treatment of the finite channel's length and of the role of elastic scattering. A quantum theory of the acoustoelectric effect in a uniform quantum channel was developed in Ref. [12] in an analogy to the previous treatment of equilibrium phonons [13]. The main point was to analyze the energy and momentum conservation laws which correspond to backscattering of the electrons by the traveling wave. It was shown that there is a cutoff in the electron-SAW interaction at $q=q_{0} \equiv 2 m s / \hbar$. At $q \leq q_{0}$ backscattering is forbidden, while the forward scattering cannot influence the current in a $1 \mathrm{D}$ system. This statement has been also expressed in a recent paper 114. where the interaction with non-equilibrium ballistic phonons was considered. At the same time, in the experiments [1]2] the interaction is clearly observed despite of the inequality $q \ll q_{0}$.

Our aim is to draw attention to the fact that in any realistic quantum channel, there are special regions near the leads where an acoustic wave (or non-equilibrium ballistic phonons) can cause transitions between the propagating states in the channel and the reflecting states which correspond to reflection of an electron back into the incident lead. These particular processes facilitate phonon-induced backscattering. As a result, the acoustoelectric effect persists at $q \leq q_{0}$ and several new and important features of the effect appear at $q \geq q_{0}$. Note that the role of the processes mentioned above was emphasized in Ref. [15] in connection with photo-conductance of quantum channels. However, there are important differences between the case of transverse electro-magnetic field with zero wave vector (as in the case of photoconductance) and the longitudinal wave with a finite wave vector (as in the case of the acoustoelectric effect). Below we present a quantum theory which takes into account the above mentioned transitions and in this way explains the results obtained in realistic structures. We believe that at $q \ll q_{0}$ these transitions lead to the same physical effect as the introduction of a finite relaxation length in the classical theory [11], while they do not have analogs in the theory for an uniform channel [12,14.

The paper is organized as follows. In section [I] we briefly go through the basic assumptions and formalism.
Section III is devoted to simple semi-classical analysis and is most relevant for high frequency fields as generated with the focused laser beam techniques. Section IV concerns the low frequency regime where semi-classical theory is invalid. This section is relevant for frequencies generated by SAW techniques.

\section{THEORY}

Being interested in the response of quasi-onedimensional systems (quantum constrictions, wires, etc.) we assume that the channel's width is of the order of the Bohr radius $a_{B}$ while the leads consist of a twodimensional electron gas and have widths $\gg a_{B}$. On the other hand, the typical wave lengths $2 \pi / q$ of SAW or non-equilibrium phonons is $\geq a_{B}$. As a result, the (piezo)electric field produced by acoustic waves is efficiently screened outside the channel. This is the reason why the response of a short channel is not completely masked by the leads. Consequently, the field is taken into account only in the region near the constriction. Apart from this, we assume that the electrons are noninteracting and spin degenerate. For simplicity we consider the case of zero temperature and assume the quantum channel to be symmetric with respect to reflection of the longitudinal coordinate $(x \rightarrow-x)$. Under such an assumption there is no so-called "rectified current", and the total dc current is due to directed propagation of the acoustic waves. In a real experimental situation there is no full symmetry of the channel. However the rectified current can be subtracted by taking the average of the results obtained by reversing the direction of SAW (or phonon) propagation. Let the channel be elongated along the $\mathbf{x}$-axis, its dimensions in the $\mathbf{y}$ - and $\mathbf{z}$-directions being much less than $q_{y}^{-1}$ and $q_{z}^{-1}$, respectively. In that case non-equilibrium phonons cannot produce transitions between the modes of transversal electron motion. Consequently, all transverse modes are subjected to the sliding potential

$$
V(x, t)=V(x) \cos (q x-\omega t), \quad q \equiv q_{x},
$$

since under the assumptions made above the envelope function $V(x)$ is independent of the transverse mode number 16].

Neglecting spontaneous emission in the constriction region, we can write the dc electric current from left $(\mathrm{L})$ to right $(\mathrm{R})$ as [17. 18]

$$
\begin{aligned}
I=\frac{2 e}{h} \sum_{n} \int_{0}^{\infty}\left[T_{R, L}(E+n \hbar \omega, E) f_{\mu_{L}}(E)-\right. \\
\\
\left.T_{L, R}(E+n \hbar \omega, E) f_{\mu_{R}}(E)\right] \mathrm{d} E .
\end{aligned}
$$

Here $e$ is the electronic charge while $T_{R, L}(E+n \hbar \omega, E)$ is the sum (over the transverse modes) of transmission 
probabilities for particles with the energy $E$ to be transfered from the left to the right lead into states with the final energy $E+n \hbar \omega . T_{L, R}(E+n \hbar \omega, E)$ denotes the reverse transition, both transitions being accompanied by absorption or (induced) emission of $n$ phonons. Eq. (2) is actually a generalization of the Landauer-Büttiker formula [19].

Since we are going to study dc response in the absence of external bias, i.e. at $\mu_{L}=\mu_{R}=\mu$, the current can be expressed as

$$
I(\mu)=\frac{2 e}{h} \int_{0}^{\infty} \Delta T(E) f_{\mu}(E) \mathrm{d} E
$$

where

$$
\Delta T=\sum_{n}\left[T_{R, L}(E+n \hbar \omega, E)-T_{L, R}(E+n \hbar \omega, E)\right] .
$$

A positive $\Delta T(E)$ means that non-equilibrium phonons enhance the left $\rightarrow$ right $(L \rightarrow R)$ dc particle current. It will be illustrative later to analyze the behavior of $\Delta T(E)$ in terms of partial changes in the $L \rightarrow R$ and $R \rightarrow L$ transmission probabilities. We therefore define

$$
\begin{aligned}
& \Delta T_{R, L}(E)=\sum_{n} T_{R, L}(E+n \hbar \omega, E)-T^{0}(E) \\
& \Delta T_{L, R}(E)=\sum_{n} T_{L, R}(E+n \hbar \omega, E)-T^{0}(E),
\end{aligned}
$$

where $T^{0}(E)$ is the sum of transmission probabilities in the absence of acoustic perturbation.

In this work we shall study the regime of relatively small acoustic amplitudes where a perturbative approach is adequate. In particular, we restrict ourselves with the linear in acoustic intensity approximation where it is sufficient to consider $n=-1,0$ and 1 . In the following we present the numerical analysis of the induced current based on the recursive Green function method near the continuum limit [18]. This method gives an exact solution to the scattering problem posed by the Schrödinger equation. For small systems, such as those under the present consideration, super-computing facilities are not necessary. If not otherwise stated, the quantum channel consists of 2DEG, and its shape is chosen in the form

$$
\frac{W(x)}{W_{\infty}}=\frac{W_{0}}{\left(W_{\infty}-W_{0}\right) \cos ^{4}(\pi x / 2 L)+W_{0}} .
$$

Here $W_{\infty}$ is the width in the region of the leads, $W_{0}$ is the minimal width, while $L$ is the characteristic length. The envelope function, $V(x)$, in equation (1) is chosen as

$$
V(x)=V_{0}\left[1-\sin ^{8}(\pi x / 4 L)\right],
$$

when $|x|<2 L$ and zero otherwise. The square-box confining potential in the $\mathbf{y}$-direction is chosen such that the transverse wave functions vanish at $y= \pm W(x) / 2$. For the calculations presented we have chosen $W_{\infty}=8 W_{0} \simeq$ $0.3 \mu \mathrm{m}$ and $k_{F} \simeq 1.25 \times 10^{8} \mathrm{~m}^{-1}$. The sound velocity is set to $s=2700 \mathrm{~m} / \mathrm{s}$, and the effective mass $m^{*}=$ $0.067 m_{e}$, typical for AlGaAs-structures.

The general behavior of $\Delta T(E)$ appears rather complicated. However, as it will be shown below, there are limiting cases of low and high frequencies where the basic features can be understood from very simple arguments. We give these arguments below.

\section{HIGH FREQUENCY REGIME}

In the high-frequency regime it is natural to consider the acoustically induced effects as scattering of electrons by non-equilibrium phonons. For simplicity, let us consider a constriction with smooth variations on the length scale set by the Fermi wavelength $k_{F}^{-1}$. In this case we can use an adiabatic and semi-classical analysis [20]. The essence of the adiabatic approximation is to assume the electronic wave function can be expressed as a product of the transverse part (which varies slowly as a function of the longitudinal co-ordinate $x$ ), and the longitudinal part which bears the main $x$-dependence. Consequently, the shape of the constriction manifests itself only as rather smooth $x$-dependence of the transverse eigen energies, $E_{m}(x)$, for different transverse modes. This $x$-dependent eigen energy, $E_{m}(x)$, will now act as a potential barrier for an one-dimensional scattering problem describing electron transfer in the $x$-direction. We put the origin of the reference frame in $x$-direction at the maximum of $E_{m}(x)$ where the constriction is the narrowest.

The semi-classical approximation ignores quantum mechanical tunneling. This implies that the zero field transmission probability, $T^{0}(E)$ for the $m$ th mode, is zero when $E<E_{m}(0)$ (the reflecting region) and one when $E>E_{m}(0)$ (the propagating region). The range of applicability of the semi-classical approximation is determined by the energy window, $\delta E$, where $T^{0}(E)$ goes from zero to one. A sufficient criterion is $\hbar \omega \gg \delta E$.

An important feature of the semi-classical situation is that the phonon-assisted processes are spatially confined to regions relatively small in comparison to the total length of the channel, $L$. Indeed, in complete analogy with the semi-classical theory of photo-conductance [15], only in the vicinities of the points $x^{*}$, corresponding to local conservation for both momentum and energy, the phase of the integrand which enters the perturbation matrix element $\langle i|V(x, t)| f\rangle$ is a slow function of $x$. Consequently, only the vicinities of such points are important, and the general behavior of the induced current can be understood from the analysis of the local conservation laws.

In the semi-classical approximation, the longitudinal part of the wave function can be expressed as 


$$
\psi_{\|}=\sqrt{\frac{k(\infty)}{k(x)}} \begin{cases}\exp \left(\imath \int^{x} k\left(x^{\prime}\right) d x\right), & E>E_{m}(0) \\ \sin \left(\int^{x} k\left(x^{\prime}\right) d x\right), & E<E_{m}(0)\end{cases}
$$

where the local longitudinal momentum is defined as

$$
\hbar k(x, E)=\sqrt{2 m^{*}\left(E-E_{m}(x)\right)} .
$$

From now on, assume that the sliding potential in equation (1) is moving from the left to the right (i.e. $q>0$ ). Conservation of energy and local longitudinal momentum implies that intra-band transitions can only take place between two states (labeled 1 and 2) at points $x^{*}$ when

$$
k_{1}\left(x^{*}, E\right)=\frac{q_{0}-q}{2}, k_{2}\left(x^{*}, E+\hbar \omega\right)=\frac{q_{0}+q}{2},
$$

where $q_{0} \equiv 2 m s / \hbar, m$ is the effective mass, $s$ is the sound velocity. Here we have taken into account that for acoustical phonons $\omega=s q$. Note that the component $\propto \exp (i q x-i \omega t)$ in $V(x, t)$ will cause a transition from the state 1 to the state 2 (absorption), while the component $\propto \exp (i \omega t-i q x)$ will cause a transition from the state 2 to the state 1 (emission). One can observe the characteristic quantity $q_{0}$ which sets the scale for the phonon wave vector $q$.

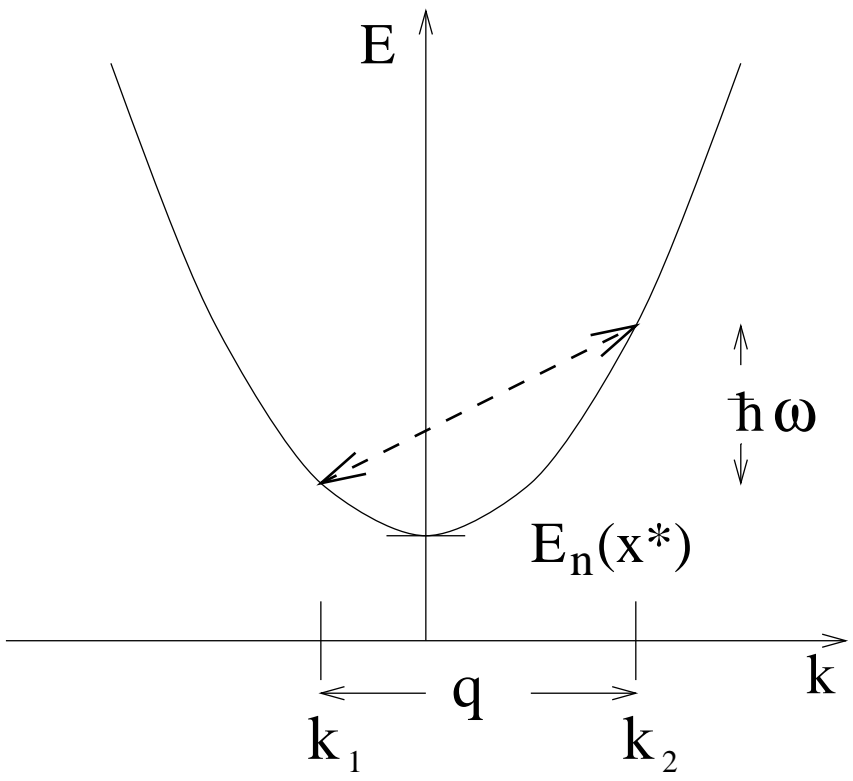

FIG. 1. Transitions that satisfy both conservation of momentum and energy

Consider a long channel with an uniform width such as was considered in 12,14. In such a channel, only scattering between states propagating in opposite directions can contribute both to the drag current and to the acoustoconductance. This implies that we must have $q>q_{0}$ in order to see any effect. However, for non-uniform wires this is no longer the case. As it follows from Fig. 11 and Eqs. (11), it is natural to analyze the cases $q<q_{0}$ and $q>q_{0}$ separately.
The case $q<q_{0}$

The case $q<q_{0}$ is the simplest. Although the criterion $\hbar \omega \gg \delta E$ for the semi-classical treatment is usually not met at $q<q_{0}$ in realistic systems, this approach is very illustrative and leads to qualitatively correct answers. This is why it has recently been used to study the acoustoelectric current [12] and acousto-conductivity 114 in quantum wires. The authors concluded that neither current nor the change in the conductance should be induced at $q<q_{0}$.

However, the co-ordinate dependence of the channel width leads to a finite backscattering even at $q<q_{0}$. If the energy $E$ of the initial state satisfies the condition

$$
E_{m}(0)-\hbar \omega<E<E_{m}(0)
$$

then a scattering from the initial reflecting state (incoming from the left) with the energy $E$ to the final propagating (to the right) state with the energy $E+\hbar \omega$ is allowed. As a result of the absorption process described above, the initial state becomes not fully reflecting, and the total current through the channel increases. An emission process from the energy $E$ to the energy $E-\hbar \omega$ will not influence the total transmission. On the other hand, when

$$
E_{m}(0)<E<E_{m}(0)+\hbar \omega
$$

an emission process will reduce $\Delta T_{R, L}(E)$ while absorption is not important. Both situations are illustrated in Fig. 2 (panels a and b, respectively). When $E>$ $E_{m}(0)+\hbar \omega$, no transitions will alter the transport properties since transitions are between states fully transmitted in the same direction. Fig. 3 is a sketch of the resulting $\Delta T(E)$ and the DC response, $I(\mu)$. The presented qualitative picture is confirmed by the numerical calculations. We do not present them in detail because the requirement $q<q_{0}$ can be hardly fulfilled together with the criterion $\hbar \omega \gg \delta E$ in realistic structures.

We believe that the effect is qualitatively similar at $\hbar \omega \lesssim \delta E$ (where the semi-classical approach is not valid), but less pronounced. In fact, the DC response is very similar even when $\hbar \omega \ll \delta E$ (as will be shown in the next section), although the physics is quite different. 
a)

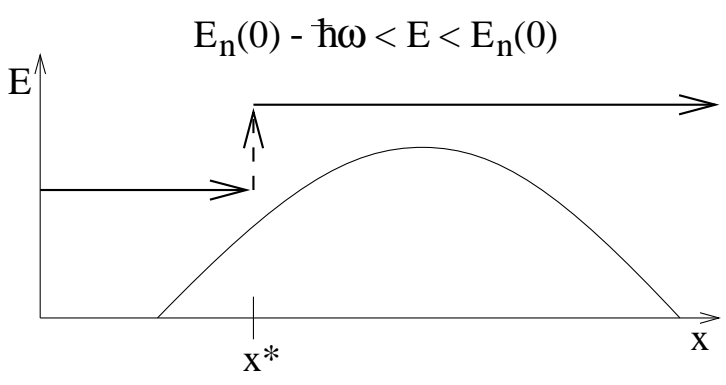

b)

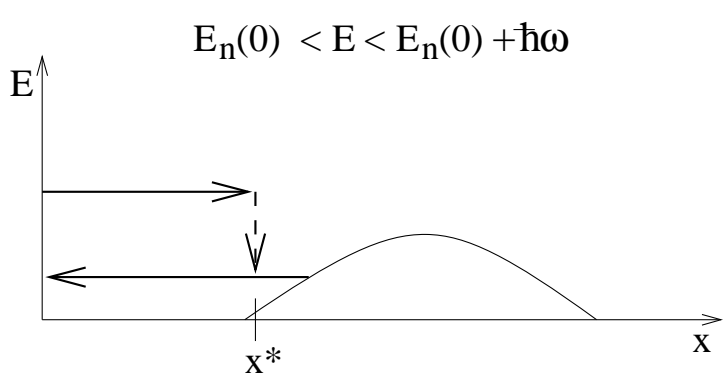

FIG. 2. A schematic view of the inelastic scattering processes which give rise to induced direct current when $q<q_{0}$.

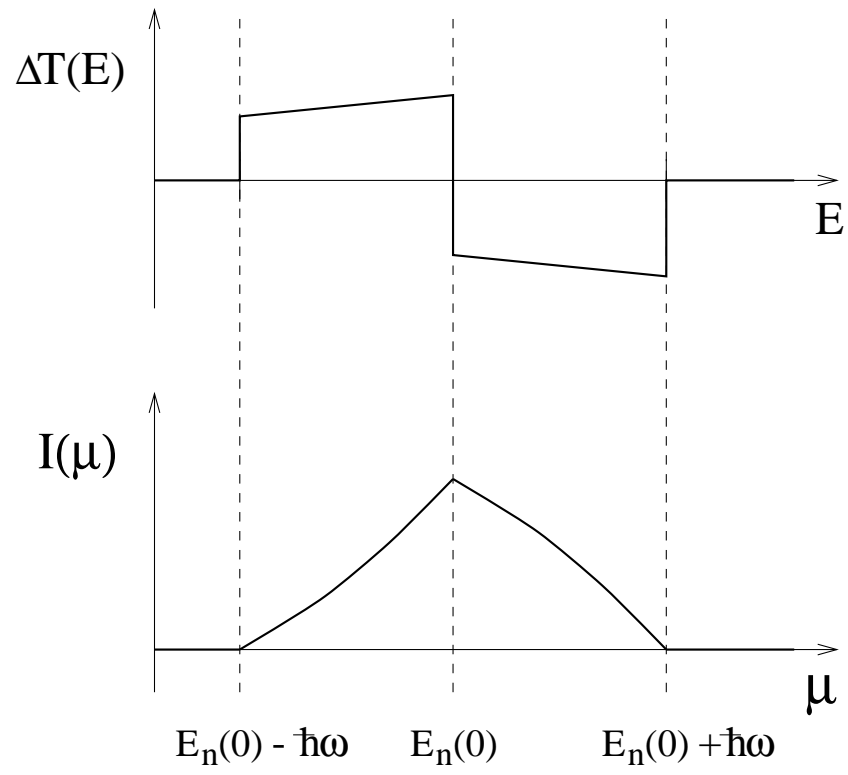

FIG. 3. A schematic drawing of the net transmission probability, $\Delta T(E)$, and the induced current $I(\mu)$ when $q<q_{0}$.

The case $q>q_{0}$

For the case when $q>q_{0}$ things are slightly more complicated since from equation (11) scattering from the acoustic field will now reverse the direction of propagation. From the conservation laws (11) one can extract the relevant energy scale,

$$
\epsilon_{q}=\hbar^{2}\left(q+q_{0}\right)^{2} / 8 m
$$

If $\epsilon \equiv E-E_{m}(0)>\epsilon_{q}$ then no scattering is possible which fulfills conservation of both energy and local longitudinal momentum. One can discriminate between the following four energy intervals for the energy $\epsilon$.

In the first one (see Fig. 田, a),

$$
-\hbar \omega<\epsilon<0,
$$

the wave with the energy $E$, incoming from the left lead and initially reflected, absorbs the energy $\hbar \omega$ and momentum $\hbar q$, the final state being propagating. The process results in a positive $\Delta T(E)$ because in this energy interval $\Delta T_{L, R}(E)$ is not affected by inelastic scattering.

In the second region, (see Fig. 国, b),

$$
0<\epsilon<\epsilon_{q}-\hbar \omega,
$$

the waves incoming from both the left and the right leads will experience scattering which affects $\Delta T(E)$. The electrons, incoming from the left, may emit energy $\hbar \omega$ and momentum $-\hbar q$ and then be reflected. Such a process takes place in the vicinity of the point $x_{L}^{*}$ where the conservation laws are met. This results in a negative $\Delta T_{R, L}$. On the other hand, the particles incoming from the right may absorb an energy quantum. It is important that there are two points, $x_{R}^{*}$ and $-x_{R}^{*}$ where the conservation laws are met. Near both points a backscattering takes place, therefore the contributions to $\Delta T_{L, R}(E)$ are also negative. The fact that we have two scattering points has the following implications:

- The resulting state is a superposition of an incoming wave and two reflected waves, the reflecting points being $\pm x_{R}^{*}$. Thus the dependence of $\Delta T_{L, R}$ vs. energy (actually, vs. the Fermi level controlled by the gate voltage) shows oscillations due to interference effects between the reflected waves.

- The amplitude of oscillations in $\Delta T_{L, R}(E)$ is about four times greater than $\Delta T_{L, R}(E)$ for a single scattering event. Thus in the above mentioned region the interference scattering dominates $\Delta T(E)$.

In the third region (Fig. 囵,

$$
\epsilon_{q}-\hbar \omega<\epsilon<\hbar \omega
$$

there is only one point where the scattering takes place. Consequently, the interference phenomena described above do not occur. Here, the only effect is inelastic backscattering of the electrons incoming from the left via emission of a phonon. Hence, $\Delta T(E)<0$ in this region.

The fourth region (Fig. 1 ,d) is defined as

$$
\hbar \omega<\epsilon<\epsilon_{q}
$$


In this region there is still backscattering of states incoming from the left, but now there are two scattering points giving rise to an interference phenomena similar to that described above for the second region. As a result, $\Delta T(E)$ is negative and oscillating with a relatively large amplitude.

(a)

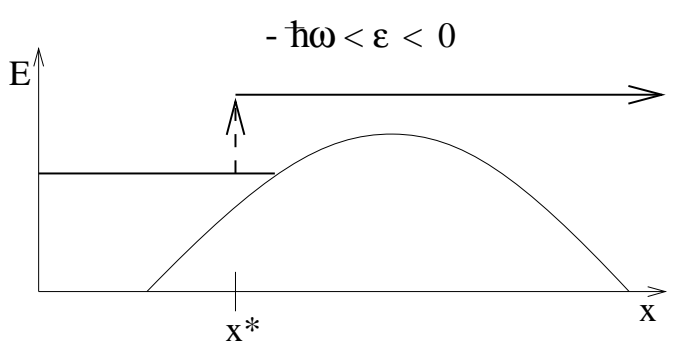

(b)

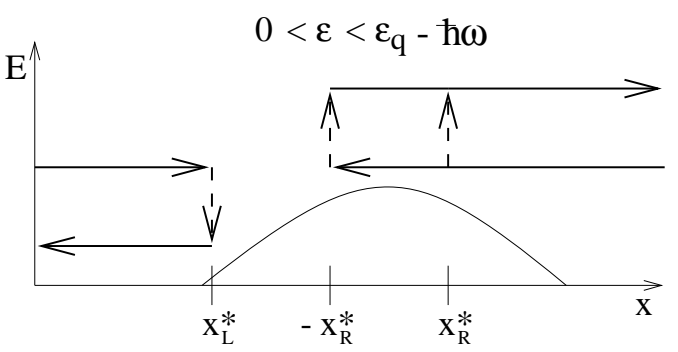

(c)

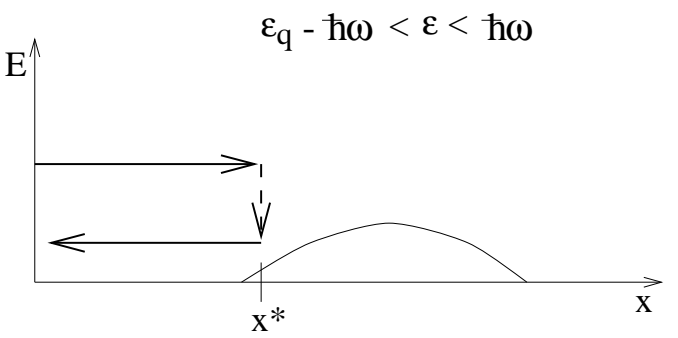

(d)

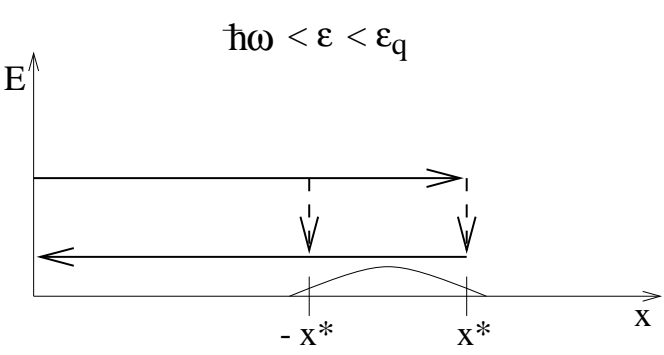

FIG. 4. A schematic illustration of the different scattering mechanisms which influence the induced direct current when $q>q_{0}$.

From the given simple analysis we observe that when $q>(3+2 \sqrt{2}) q_{0}$ the third region vanishes, and there are interference oscillations for all the energies in the interval

$$
0<\epsilon<\epsilon_{q}
$$

Such a situation seems typical for the phonon-dragimaging experiments where $\omega / 2 \pi$ can exceed $100 \mathrm{GHz}$. 99
Note that at $q \gg q_{0}$ the sufficient condition to employ the semi-classical approximation is $\max \left\{\epsilon_{q}, \hbar \omega\right\} \gg \delta E$ which is weaker than $\hbar \omega \gg \delta E$. In addition, one must require $q L \gg 1$ in order to have any interference.

a)

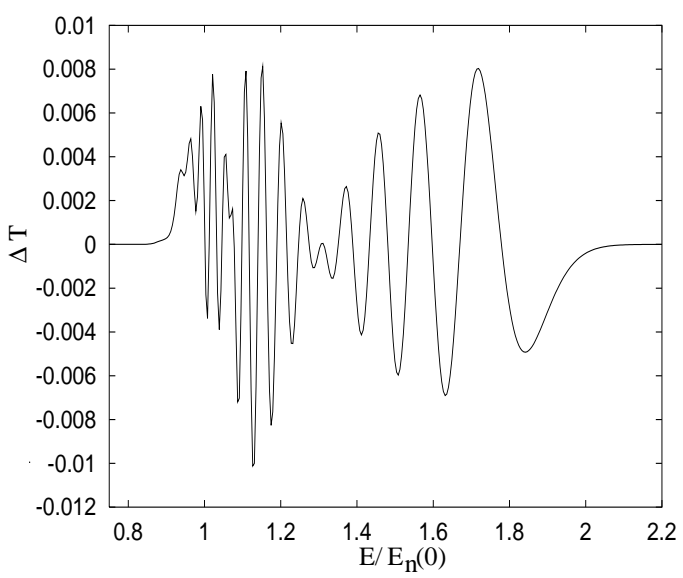

b)

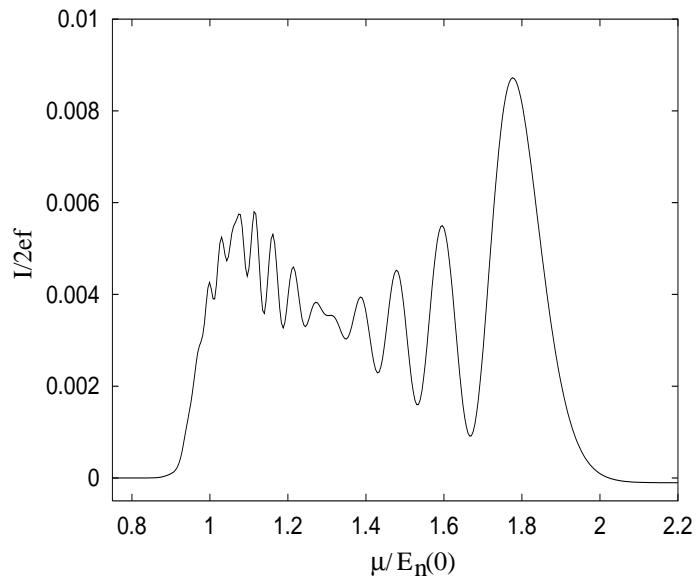

FIG. 5. The effect of the phonon scattering when $q=50 q_{0}$. The inner length of the constriction is $l \simeq 1 \mu \mathrm{m}$. a) The induced $\Delta T(E)$. b) The induced dimension-less current, $I / 2 e f$, plotted as a function of the chemical potential, $\mu$. The interference oscillations are clearly seen in the induced current.

Figures 5 and 6 show the results of numerical calculations for $q=50 q_{0}$. Fig. 6 refers to a total length of the constriction region of $2 L=2.4 \mu \mathrm{m}$ [see equation (7)], which yields an inner constriction length, $l$, of approximately $1 \mu \mathrm{m}$. Fig. 5, a shows $\Delta T(E)$ as defined in equation (4) while Fig. 6, b shows the induced dimensionless current, $I / 2 e f$, as a function of the chemical potential. The interference effects are quite pronounced, even for the induced current. Fig. 6 shows the results of the numerical calculations when $2 L=0.6 \mu \mathrm{m}$ which means that the length of the inner region is approximately $l=0.25$ $\mu \mathrm{m}$. Panel a shows the zero field transmission. In addition, vertical lines indicating the position of the energies $E_{m}(0) \pm \hbar \omega$ are displayed. Clearly, in this case $\hbar \omega \lesssim \delta E$. However, as it is seen in the panel b, the interference effects in the induced current are still visible.

In an experimental situation with non-equilibrium 
phonons generated by a point-like heater the phonons are not monochromatic. To observe interference effects in such a situation, one needs to meet the inequality $\Delta \omega L / s \lesssim 1$, where $\Delta \omega$ is the characteristic width of the phonon frequency distribution. This requirement implies $\Delta \omega / \omega \ll 1$.

a)

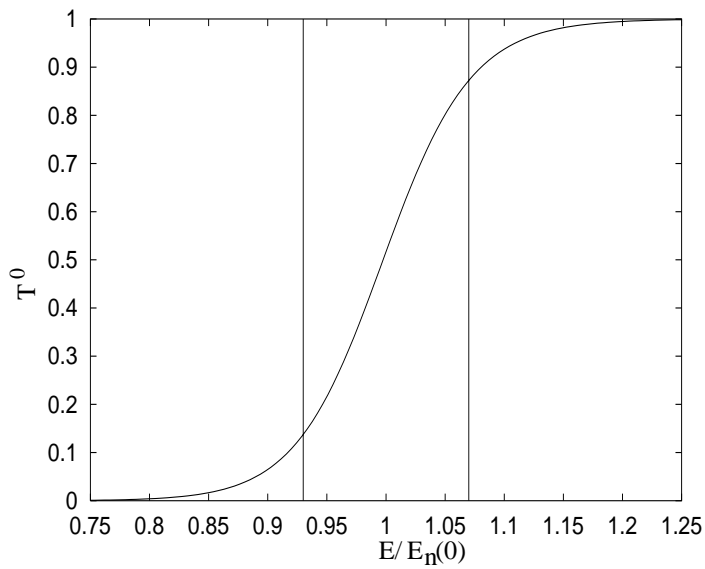

b)

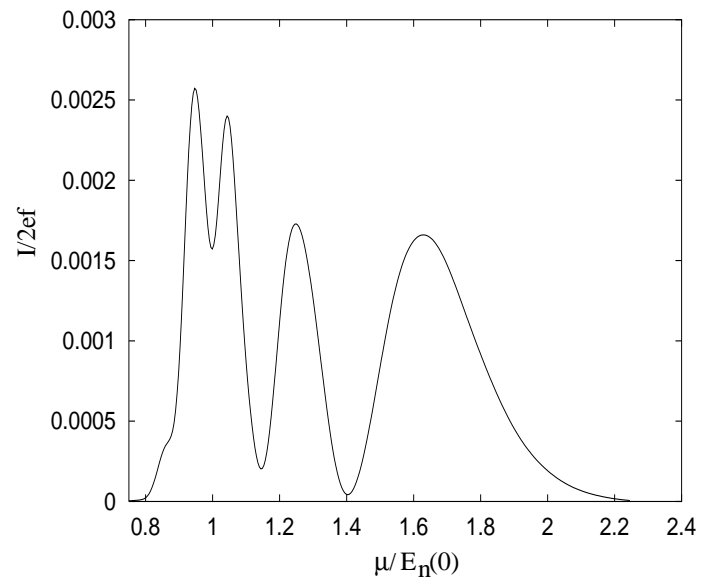

FIG. 6. The effect of the phonon scattering when $q=50 q_{0}$. The inner length of the constriction is $l \simeq 0.25 \mu \mathrm{m}$. a) The zero field transmission, $T^{0}(E)$. The vertical lines indicate $E_{n}(0) \pm \hbar \omega$. Notice that $\hbar \omega<\delta E$. b) The induced dimensionless current, $I / 2 e f$, plotted as a function of the chemical potential, $\mu$.

\section{LOW FREQUENCY REGIME}

So far, we have studied the semi-classical regime where the transition region for $T^{0}(E), \delta E$, is much smaller than $\hbar \omega$. However, in many experiments using coherent SAWs [1] to induce the current, this is not the case. We should therefore investigate what happens with the acoustoelectric current at low frequencies, in particular, when $\hbar \omega \ll \delta E$.

Suppose that the time dependence of the SAWpotential can be considered as adiabatic in time, i.e $\omega / 2 \pi \ll v_{F} / L$ (or $q L \ll 2 \pi v_{F} / s$ ). Let us also assume that the SAW wavelength is large compared to the con- striction length $(q L<2 \pi)$. In the limit of infinite wavelength we must have $\Delta T_{R, L}=\Delta T_{L, R}$ since the symmetry $x \leftrightarrow-x$ is not broken any more. To a first approximation we should then have

$$
\begin{aligned}
\Delta T_{R, L}(E) & \simeq \Delta T_{L, R}(E) \\
& \simeq \frac{\omega}{2 \pi} \int_{0}^{2 \pi / \omega} T^{0}\left[E+V_{0} \cos (\omega t)\right] \mathrm{d} t-T^{0}(E) \\
& \simeq \frac{V_{0}^{2}}{4} \partial_{E}^{2} T^{0}(E) .
\end{aligned}
$$

It should be emphasized that in this regime there is no distinction between the cases $q<q_{0}$ and $q>q_{0}$. Fig. 7, a displays $\Delta T_{R, L}(E)$ and $\Delta T_{L, R}(E)$ together with the theoretical prediction, equation (17). Note that there are no parameters to fit. The results in Fig. 7 were obtained with $q=3 q_{0}$ and are also representative for the case $q<q_{0}$.

a)

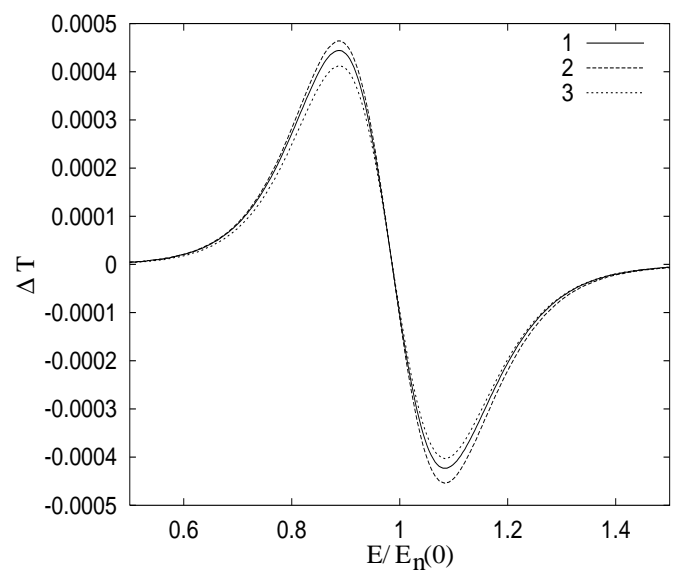

b)

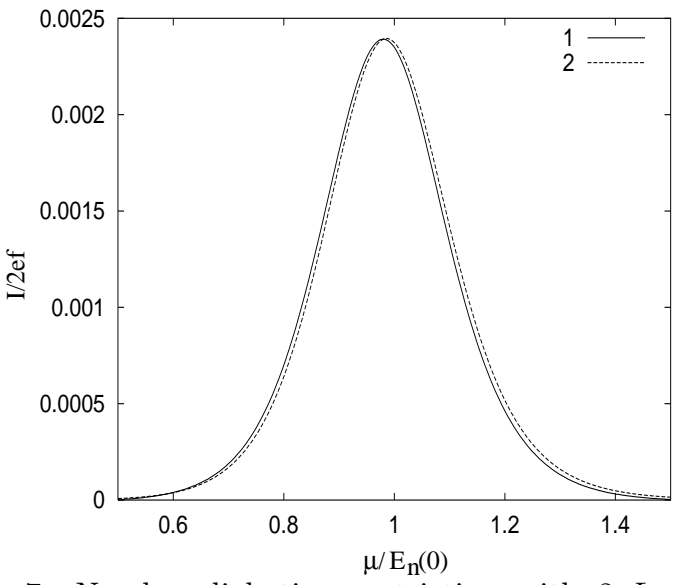

FIG. 7. Nearly adiabatic constriction with $2 q L=2.8$ $\left(q=3 \times q_{0}\right)$. a) Curve 1 shows $V_{0}^{2} \partial_{E}^{2} T^{0}(E) / 4$, curve 2 shows $\Delta T_{R, L}(E)$ and curve 3 shows $\Delta T_{L, R}(E)$. There are no parameters fitted. b) Comparison of the dimensionless current, $I(\mu) / 2$ ef (line 1), and $\partial_{E} T^{0}(E) \times \hbar \omega$ (line 2). Line 2 is scaled to match the amplitude of line 1.

In general there is also some dependence on finite $q$, which is the origin of the induced current. One can ex- 
pect that such a dependence at small $q$ is proportional to $q \omega$ because the effect must be odd in $q$ and proportional to $\omega^{2}$ at small frequencies. The effect of broken symmetry (finite $q$ ) can be clearly seen in Fig. 7, a which shows that $\left|\Delta T_{R, L}(E)\right| \geq\left|\Delta T_{L, R}(E)\right|$. This yields the induced dc current which is plotted in Fig. 8,b together with $\hbar \omega \partial_{\mu} T^{0}(\mu)$. The curve representing $\hbar \omega \partial_{\mu} T^{0}(\mu)$ is scaled as to match the amplitude of the dimension-less current, I/2ef. For smoothly varying constrictions, the numerical calculations show that

$$
\Delta T_{R, L}(E)-\Delta T_{L, R}(E) \simeq g \frac{V_{0}^{2}}{4 s} q \omega L^{2} \partial_{E}^{2} T(E),
$$

when $q L / 2 \pi<1, v_{F} / s$. The dimensional factor $g$ in (18) is geometry dependent and almost $L$-independent. This yields the current

$$
I(\mu) \simeq g \frac{e V_{0}^{2}}{2 s h} q \omega L^{2} \partial_{\mu} T^{0}(\mu),
$$

which explains the strong resemblance of the induced current and $\partial_{\mu} T^{0}(\mu)$. The similar behavior of the induced current and $\partial_{\mu} T^{0}(\mu)$ agrees with the experimental findings [1]. In an experiment, one alters the gate voltage, $V_{g}$, rather than the chemical potential. Changes in $V_{g}$ alters both the geometry of the constriction (width and length) and the electron density within the constriction. Since screening effects are dependent on the electron density, both the effect of impurities and the effective coupling of the induced field to the electrons are dependent on the electron density. However, within small regions of the gate voltage, the effect of changing $V_{g}$ should be very similar to changes in $\mu$.

So far we have studied the regime of weak elastic intermode scattering. It is natural to ask what happens in the presence of strong elastic inter-mode scattering. To investigate this we have considered an abrupt channel with

$$
W(x)=\left\{\begin{array}{ll}
W_{0} & \text { at }|x|<L / 2 \\
8 W_{0} & \text { at }|x|>L / 2
\end{array} .\right.
$$

Fig. 8,a shows $\Delta T_{R, L}(E)$ and $\Delta T_{L, R}(E)$ together with the result of Eq. (17). As can be seen, Eq. (17) still holds. The induced current is displayed in Fig. 8. b together with $\partial_{\mu} T^{0}(\mu)$. It seems obvious that Eq. (19) no longer holds, although there are some similarities between the two curves. In particular, the scaling of the induced current, $I \sim q \omega$, is remarkably well satisfied. This is shown in Fig. 9. We would like to emphasize once more that $I \sim q \omega$ and not $I \sim \omega^{2}$ nor $I \sim q^{2}$. This fact has been checked explicitly by an independent choice of $q$ and $\omega$. It is also in agreement with the recent work [11] based on the Boltzmann equation. The $L^{2}$-dependence for the induced current for long enough waves was also found in [11], according to [14] such a dependence holds also for acousto-conductivity. a)

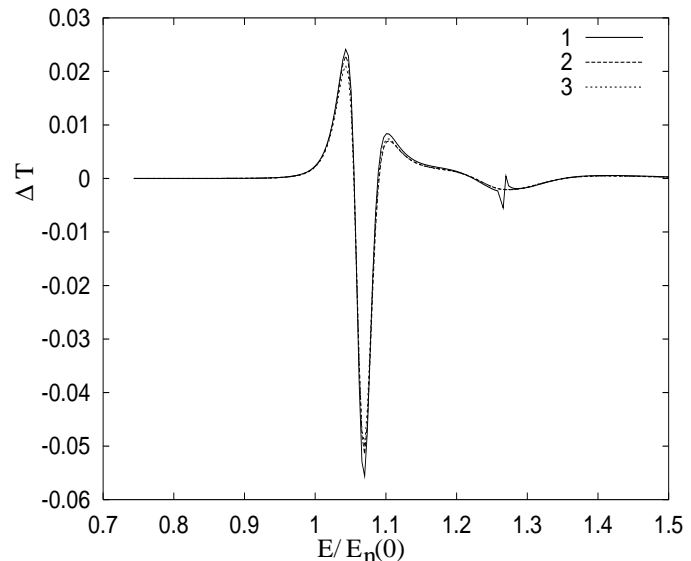

b)

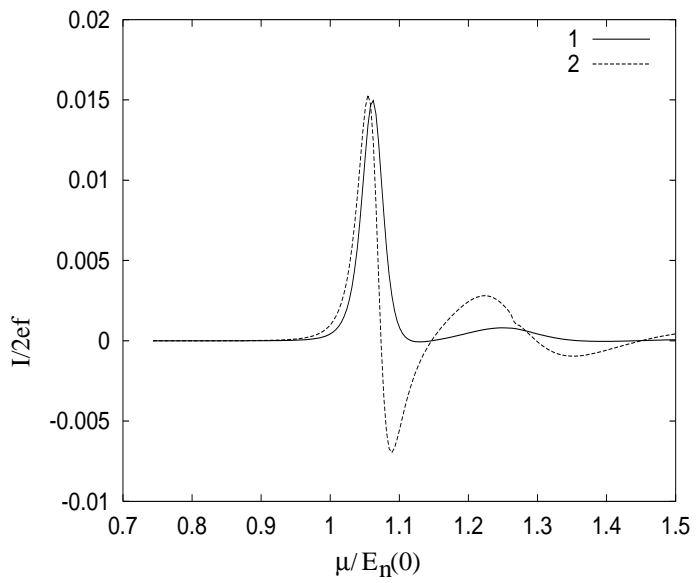

FIG. 8. Abrupt constriction with strong elastic scattering when $2 q L=1.12\left(q=3 \times q_{0}\right)$. a) Line 1 shows $V_{0}^{2} \partial_{E}^{2} T^{0}(E) / 4$, line 2 shows $\Delta T_{R, L}(E)$ and line 3 shows $\Delta T_{L, R}(E)$. There are no parameters fitted. The kink in line 1 is an artifact caused by the sudden appearance of the 9 th transverse state. b) Comparison of the dimension-less current, $I(\mu) / 2$ ef (line 1 ), and $\partial_{E} T^{0}(E) \times \hbar \omega$ (line 2). The amplitude of line 2 is scaled to match the amplitude of line 1 .

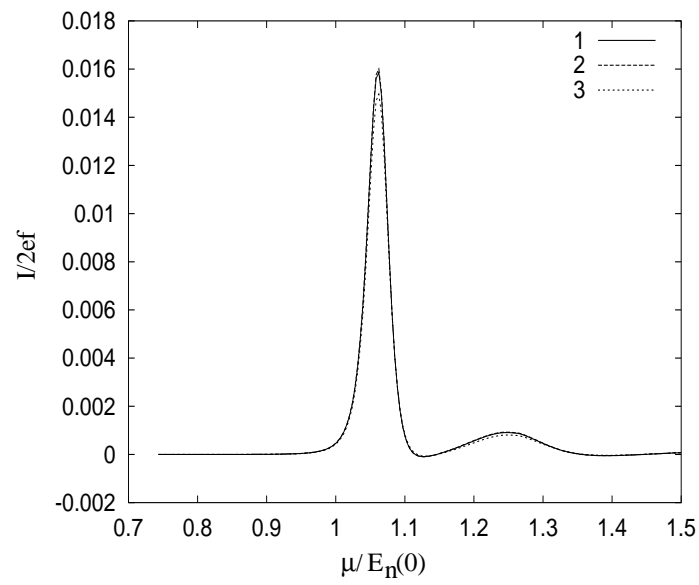

FIG. 9. Comparison of the dimensionless current, $I(\mu) / 2 e f$, times $3 q_{0} / q$ for an abrupt constriction when $q=q_{0} / 2$ (line 1), $q=q_{0} \sqrt{6} / 2$ (line 2) and $q=3 q_{0}$ (line 3). The figure suggests that $I \sim q \omega$. 
Eq. (17) gives the acousto-conductance, $\Delta G$. Since the numerical results agree so well with Eq. (17), it is tempting to speculate about the second harmonic of the ac current. It seems most advantageous to study the second harmonic at low enough frequencies $(q L \ll 2 \pi)$ applying a small dc bias voltage, $V_{b}$. In such a situation, in addition to the dc current (19) and the dc Ohmic current proportional to $V_{b}, I_{0}=2\left(e^{2} / h\right) T^{0}(\mu) V_{b}$, there will also be the ac current at frequency $2 \omega$ which is also proportional to $V_{b}$. This current is originated by ac modulation of the transparency $\Delta T(E)$. According to Eq. (17) this current is simply given by

$$
I_{2 \omega} \simeq \frac{e^{2} V_{0}^{2}}{2 h} \cos (2 \omega t) \partial_{\mu}^{2} T^{0}(\mu) V_{b},
$$

Thus, the dc acousto-conductance and the "second harmonic conductance", $I_{2 \omega} / V_{b}$, are the same. Furthermore,

$$
\frac{I_{2 \omega}}{I_{0}}=\frac{\Delta G}{G_{0}} \cos 2 \omega t=\frac{V_{0}^{2}}{4} \frac{\partial_{\mu}^{2} T^{0}(\mu)}{T^{0}(\mu)} \cos 2 \omega t .
$$

Having measured the dependence $T^{0}(\mu)$ from the $I-V_{b}$ curve, one can estimate the actual amplitude $V_{0}$ of the acoustically-induced potential. The second harmonic ac current may be also important from an experimental point of view, because the main harmonic ac signal may be masked by the electrical signal from the transducer which produces SAW. 21]

\section{SUMMARY AND DISCUSSION}

We have investigated transport properties of quantum constrictions under the influence of a traveling timedependent potential created either by a coherent surface acoustic wave, or by non-equilibrium phonons. The results of numerical analysis based on the recursive Green function technique are explained in the framework of a qualitative picture of phonon-induced transitions between the electronic states.

The main result is that the dc acoustoelectric current induced in the channel is crucially dependent on the (unavoidable) transitions between propagating and reflecting states. It is those transitions that keep the current finite at $q \leq 2 \mathrm{~ms} / \hbar$ in contrast with the prediction of the paper [12] where only the transitions between propagating states were considered. In a smooth enough channel one can localize the important transition in space. It appears that at high frequency, $q \geq 2 m s / \hbar$, there are several regions where the transitions take place. As a result, an interference pattern in the dependence of the acoustoelectric current on the gate voltage is predicted. The pattern can be observed for rather monochromatic phonon distributions, criteria being discussed. Note that impurity potentials in the channel can also lead to a phonon-induced backscattering and to interference patterns.
The set of above mentioned results is valid for the channels with sharp steps of conductance quantization, where the width, $\delta E$, of the energy region (where the semi-classical approach is not valid) is less than $\hbar \omega$. We expect that the effect of finite temperature is the same as for finite $\delta E$. It is demonstrated that the acoustoelectric current exists also at $\hbar \omega \ll \delta E$ provided $q L \ll 2 \pi$. It is proportional $q \omega L^{2}$, as well as to the derivative of the transmission probability with respect to the Fermi level. The role of strong elastic scattering due to abrupt shape of the channel is studied. It is shown that the effect persists, and it is also proportional to $q \omega$ at low frequencies.

For low frequencies, also the acousto-conductance is studied. In particular, when $q L \ll 2 \pi$ the explicit formula (17) for the change in transmission is shown to hold even in the case of strong elastic scattering. It is speculated that this approach can also predict the ac current at frequency $2 \omega$ through a biased channel.

In conclusion, we want to emphasize that the drag of electrons in quantum constrictions by a sliding potential produced by SAWs or non-equilibrium phonons is a rich phenomenon which involves quantum transitions of different types. In contrast to the prediction of the paper [14] (where only the transitions between propagating modes were considered), we observe that the transitions between the propagating and reflecting states can both decrease and increase transmission probability and thus lead both to negative or positive acousto-conductance. For a detailed comparison with the experimental results one needs more information about experimental dependencies of the effect on the frequency, temperature, as well as on the length and the shape of the channel.

\section{ACKNOWLEDGMENTS}

One of the authors, FAM, would like to acknowledge financial support from Norges Forskningsråd.

[1] J. M. Shilton, D. R. Mace, V. I. Talyanskii, Yu. Galperin, M. Y. Simmons, M. Pepper, and D. A. Ritchie, J. Phys.: Condens. Matter 8, L337 (1996).

[2] J. M. Shilton, V. I. Talyanskii, M. Pepper, D. A. Ritchie, J. E. F. Frost, C. J. B. Ford, C. G. Smith and G. A. C. Jones, J. Phys.: Condens. Matter 8, L531 (1996).

[3] G. R.Nash, S. J. Bending, Y. Kershaw, K. Eberl, P. Grambow and K. von Klitzing, Surf. Sci. 361/362, 668 (1996).

[4] A. J. Naylor, K. R. Strickland, A. J. Kent, and M. Henini, Surf. Sci. 361/362, 668 (1996).

[5] A. L. Efros, Yu. M. Galperin, Phys. Rev. Lett., 64, 
1959 (1990); A. Knäbchen, Y. Levinson, and O. EntinWohlman, preprint cond-mat/9604137.

[6] A. Wixforth, J. P. Kotthaus, and G. Weinman, Phys. Rev. Lett. 56, 2104 (1986); A. Wixforth, J. Scriba, M. Wassermeier, J. P. Kotthaus, G. Weinman, and W. Schlapp, Phys. Rev. B40, 7874 (1989); R. L. Willett, M. A. Paalanen, R. R. Ruel, K. W. West, L. N. Pfeiffer, and D. J. Bishop, Phys. Rev. Lett. 65, 112 (1990); R. L. Willett, R. R. Ruel, K. W. West, and L. N. Pfeiffer, Phys. Rev. Lett. 71, 3846 (1993).

[7] A. Esslinger, A. Wixforth, R. W. Winkler, J. P. Kotthaus, H. Nickel, W. Schlapp, and R. Losch, Solid State Commun. 84, 949 (1992); J. M. Shilton, D. R. Mace, V. I. Talyanskii, M. Pepper, M. Y. Simmons, A. C. Churchill, and D. A. Ritchie, Phys. Rev. B51, 14770 (1995); J. Phys.: Condens. Matter 7, 7675 (1995).

[8] L. J. Challis, A. J. Kent, and V. W. Rampton, Semicond. Sci. Technol. 5, 1179 (1990); A. J. Kent, Physica B 169, 356 (1991); D. J. McKitterick, A. Shik, A. J. Kent, and M. Heinini, Phys. Rev. B 49, 2585 (1996).

[9] H. Karl, W. Dietsche, A. Fischer, and K. Ploog, Phys. Rev. Lett. 61, 2360 (1996).

[10] It seems very difficult to measure linear in amplitude effects of isolated mesoscopic systems because of small longitudinal size.

[11] H. Totland, and Yu. Galperin, Phys. Rev. B 54, 8814
(1996).

[12] V. L. Gurevich, V. B. Pevzner, and G. J. Iafrate, Phys. Rev. Lett 77, 3881 (1996).

[13] V. L. Gurevich, V. B. Pevzner, and K. Hess, J. Phys. Cond. Matter. 6, 8363 (1994); Phys. Rev B 51, 5219 (1995).

[14] M. Blencowe, and A. Shik, Phys. Rev. B 54, 13899 (1996).

[15] A. Grincwajg, L. Y. Gorelik, V. Z. Kleiner, and R. I. Shekter, Phys. Rev. B 52, 12168 (1995).

[16] In the general case, the envelope function is proportional to the matrix element of $\exp \left(i \mathbf{q} \mathbf{r}_{\perp}\right)$ calculated between the eigen functions $u\left(\mathbf{r}_{\perp}\right)$. At small $q_{\perp}$ these matrix elements yield 1 .

[17] Supriyo Datta, and M. P. Anantram, Phys. Rev. B 45, 13761 (1992).

[18] Frank A. Maaø, and L. Y.. Gorelik, Phys. Rev. B 53, 15 885 (1996).

[19] R. Landauer, IBM J. Res. Dev 1, 223 (1957); M. Büttiker, Phys. Rev. Lett. 57, 1761 (1986).

[20] L. I. Glazman, G. E. Lesovik, D. E. Khmelnitskii, R. I. Shekhter, JETP Lett. 48, 238 (1988).

[21] We would like to point out that in order to investigate the relations (17) and (20) one could, instead of a phonon source, introduce an oscillating signal to the gate voltage which induces a perturbation with $q=0, \omega \neq 0$. 